\section{(2) OPEN ACCESS}

\title{
Estimating the burden of the COVID-19 pandemic on mortality, life expectancy and lifespan inequality in England and Wales: a population-level analysis
}

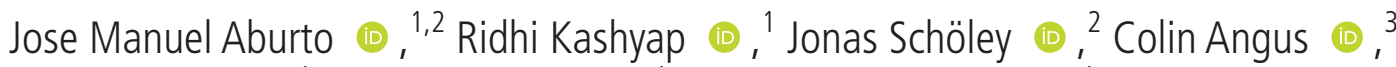 \\ John Ermisch (1) , ${ }^{1}$ Melinda C Mills (ㄷ), 'Jennifer Beam Dowd (1) ${ }^{1}$
}

\begin{abstract}
- Additional material is published online only. To view, please visit the journal online (http://dx.doi.org/10.1136/ jech-2020-215505).

'Leverhulme Centre for Demographic Science, Department of Sociology and Nuffield College, University of Oxford, Oxford, UK ${ }^{2}$ Interdisciplinary Centre on Population Dynamics, University of Southern Denmark, Odense, Denmark

${ }^{3}$ SCHARR, University of Sheffield, Sheffield, UK
\end{abstract}

\section{Correspondence to}

Dr Jose Manuel Aburto and Dr Ridhi Kashyap, Leverhulme Centre for Demographic Science, Department of Sociology and Nuffield College, University of Oxford, Oxford OX1 1JD, UK ; jose-manuel.aburto@sociology. ox.ac.uk,

ridhi.kashyap@nuffield.ox.ac.uk

Received 1 September 2020 Revised 8 December 2020 Accepted 16 December 2020 Published Online First 19 January 2021
ABSTRACT

Background Deaths directly linked to COVID-19

infection may be misclassified, and the pandemic may have indirectly affected other causes of death. To overcome these measurement challenges, we estimate the impact of the COVID-19 pandemic on mortality, life expectancy and lifespan inequality from week 10 of 2020, when the first COVID-19 death was registered, to week 47 ending 20 November 2020 in England and Wales through an analysis of excess mortality. Methods We estimated age and sex-specific excess mortality risk and deaths above a baseline adjusted for seasonality with a systematic comparison of four different models using data from the Office for National Statistics. We additionally provide estimates of life expectancy at birth and lifespan inequality defined as the SD in age at death.

Results There have been 57419 (95\% prediction interval: 54 197, 60 752) excess deaths in the first 47 weeks of $2020,55 \%$ of which occurred in men. Excess deaths increased sharply with age and men experienced elevated risks of death in all age groups. Life expectancy at birth dropped 0.9 and 1.2 years for women and men relative to the 2019 levels, respectively. Lifespan inequality also fell over the same period by 5 months for both sexes.

Conclusion Quantifying excess deaths and their impact on life expectancy at birth provide a more comprehensive picture of the burden of COVID-19 on mortality. Whether mortality will return to-or even fall below-the baseline level remains to be seen as the pandemic continues to unfold and diverse interventions are put in place.

\section{INTRODUCTION}

Estimating the number of deaths caused by the COVID-19 pandemic is an important challenge. ${ }^{1}$ Insufficient testing capacity for SARS-CoV-2, the causative pathogen of coronavirus disease, especially during the early phases of the pandemic, misclassification of causes of death and definitional inconsistencies in counting COVID-19 deaths across different sources make the true toll of COVID-19 infections hard to estimate. ${ }^{23}$ Moreover, interventions imposed during the pandemic may have indirectly affected other causes of death. ${ }^{4}$ For example, both fear of COVID-19 and the overstretching of the healthcare system may have deterred care seeking for both chronic and acute conditions, potentially increasing mortality from other, non-COVID, causes. ${ }^{5}$ Similarly, restrictions might have decreased deaths from external causes such as road traffic accidents, or increased deaths from causes such as suicide.

To overcome these measurement challenges, an alternative approach to estimate the mortality burden of COVID-19 is to quantify the number of deaths during the pandemic compared with a baseline level of what would have been expected if the pandemic had not occurred. This approach for estimating excess all-cause mortality has been widely used to quantify the mortality toll of previous epidemics such as influenza ${ }^{6}$ or $\mathrm{HIV}^{7}$ and has also begun to be applied for COVID-19. ${ }^{18}$ Excess mortality may be quantified in different ways and the "excess numbers of deaths" approach has been commonly used so far in England and Wales. ${ }^{4} 9$ While this metric provides an important measure of the burden of the pandemic on a society, simply counting total excess deaths does not provide an understanding of the substantial variation by age and sex over time in elevated mortality risks. ${ }^{10}{ }^{11}$ nor does it allow for a comparison of current mortality conditions with past conditions due to changes in population age structure over the period. Furthermore, excess deaths do not provide an understanding of the cumulative impact of the pandemic on summary indicators of population health such as life expectancy.

Life expectancy at birth is a commonly used age-standardised summary indicator of population health that expresses the average number of years a newborn would be expected to live given the death rates in a particular period. ${ }^{12}$ While no individual would actually be expected to experience these death rates throughout their life, life expectancy provides a snapshot of the mortality profile of a population in a given period. Additionally, life expectancy is a comparable indicator of population health that does not require the arbitrary choice of a standard population as done with reported standardised death rates. Furthermore, as life expectancy is sensitive to the ages at which deaths occur and because it is comparable across time, it can shed additional light on the cumulative burden of a crisis such as COVID-19 on population health and enable comparisons with previous population health conditions. Lifespan inequality is another complementary indicator of population health with implications for public health planning, which has increasingly been reported in population health research. ${ }^{13-15}$ 
While life expectancy is a measure of average mortality, lifespan inequality focuses on a second dimension of mortality: the variation in length of life between individuals in a population. It is possible for two populations to have the same life expectancy (ie, average) with different levels of lifespan inequality because of the variation in the distribution of the ages of death. Thus, lifespan inequality provides a complementary perspective that reflects how unevenly population health improvements are shared within a population, which has important implications for health and social care planning. Trends over the 20th century from high-income countries, including England and Wales, show that as life expectancy and the modal age at death have increased, lifespan inequality has tended to decrease. ${ }^{16}$ Nevertheless, the age dynamics driving improvement in each indicator is different. Reducing mortality at any age increases life expectancy. However, for lifespan inequality to decrease when life expectancy is increasing, more lives need to be saved at younger than older ages, usually below life expectancy. ${ }^{13}$ This compresses the distribution of deaths, making ages at death more similar.

We estimate all-cause excess deaths from week 10 (March 2-8), the week in which the first death attributable to COVID-19 was registered in England and Wales, to latest data that were available at the time of writing this article from week 47 of 2020 (ending 20 November). Our work builds on existing estimates and approaches in three ways. First, we provide estimates disaggregated by age and sex, to highlight variations in excess deaths during the pandemic in England and Wales. Second, we develop refined model-based counterfactual estimates of excess deaths that better account for exposures and seasonal mortality patterns. We also systematically assess the sensitivity of excess deaths to different model-based estimates. Third, we provide estimates of life expectancy and lifespan inequality during the first 47 weeks of 2020 and compare them with previous mortality trends. By considering all three measures together: excess deaths, life expectancy and lifespan inequality, this study presents a comprehensive assessment of the mortality impacts of the COVID-19 pandemic thus far.

\section{METHODS \\ Data}

We extracted all-cause death counts stratified by week of death registration and sex from 2010 to the week for which latest data were available (week 47 of 2020) from the Office for National Statistics (ONS) for England and Wales. While weekly mortality data are available by 5 -year age groups for 2020 , this level of disaggregation is not available for previous years. Therefore, we used six age-groups $(0-14,15-44,45-64,65-74,75-84$ and 85 -older years of age) for modelling weekly deaths to harmonise weekly death data across 2010-2020 and used the 5-year age intervals for calculating life expectancy and lifespan inequality estimates for 2020. We also obtained population estimates from ONS from 2010 to $2019^{17}$ and population projections for $2020 .^{18}$ As these projections represent the population at the mid-year point, we used standard interpolation techniques ${ }^{19}$ to estimate weekly mean population by sex and age groups over the study period to use them as offset in the modelling strategy. Yearly death counts by 5 -year age groups were used to calculate annual indicators ${ }^{20}$ such as life expectancy and lifespan inequality. All analyses use publicly available aggregated data. The population coverage of vital registration in England and Wales is complete. Between March and May 2020, 81.1\% of all deaths and $86.5 \%$ of deaths involving COVID-19 were registered within 1 week of occurrence. ${ }^{21}$ Death registration in this period witnessed increased efficiency compared with trends noted in previous years due to changes implemented in the Coronavirus Act $2020 .{ }^{22}{ }^{23}$ Based on trends from past years, 92\% of deaths are registered within 1 month of occurrence. As the extent of bias caused by registration delays is not properly understood, we do not attempt to implement any correction factors to minimise risks of overcorrection and inflating our findings.

\section{Excess mortality}

We estimated the baseline number of deaths in the absence of COVID-19 by fitting four different models. First, we fitted generalised additive models assuming negative binomial and Poisson distributions of deaths during the period of study. ${ }^{24}$ These models include a log-linear mortality trend by sex and age, smoothed effects for age and seasonality and an interaction between age and seasonality (see online supplemental section 1). The smoothed effects are stratified by sex. Third, we fitted a generalised Poisson linear model adjusted for year-toyear seasonality, ${ }^{25}$ also known as extended Serfling model ${ }^{26}$ (see online supplemental section 1). These previous models included indicator variables for systematic lags in death registration observed in weeks coinciding with holidays (weeks 1, 52 and 22 (bank holidays)). Finally, for our fourth model, we created a baseline by averaging the death rates observed in each week of the years 2015-2019 (see online supplemental figures 1 and 2).

We fitted the models to the weekly death counts from 4 January 2010 to the week starting on 2 March 2020. This baseline was then projected forward until 20 November 2020 (week 47). Excess mortality is then defined as the observed weekly death count minus the baseline, summed across the pandemic period from 2 March 2020 (week 10) to 20 November 2020 (week 47). From this baseline, 95\% predictive intervals were constructed by sampling death counts from negative binomial and Poisson distributions depending on the model's underlying distribution.

We report excess death estimates from the negative binomial model in the main text but estimates comparing the different approaches are provided in the online supplemental materials. This choice is based on out-of-sample predictive performance on past non-COVID weekly death counts.

\section{Demographic methods}

Life expectancy and lifespan inequality by sex were estimated using the yearly death counts and population estimates for the years preceding 2020 using standard demographic techniques, ${ }^{12}$ from which $95 \%$ predictive intervals were generated. ${ }^{27}$ For the 47 weeks of 2020 for which data were available, death counts were aggregated over age groups and death rates were calculated using a proportionally adjusted mid-year population estimate.

\section{Code and data availability}

All analyses were carried out using $\mathrm{R}$ software. ${ }^{28}$ All analysis scripts and data are available in a public repository and will be updated as more data become available. ${ }^{29}$

\section{RESULTS}

\section{Estimates of excess deaths}

The first death attributable to COVID-19 in England and Wales was registered in the week starting on 2 March 2020 (week 10). From that week until the end of week 47 on 20 November 2020, there were 436102 registered deaths, from which an estimated $57419(54197,60752)$ are excess mortality above the expected 


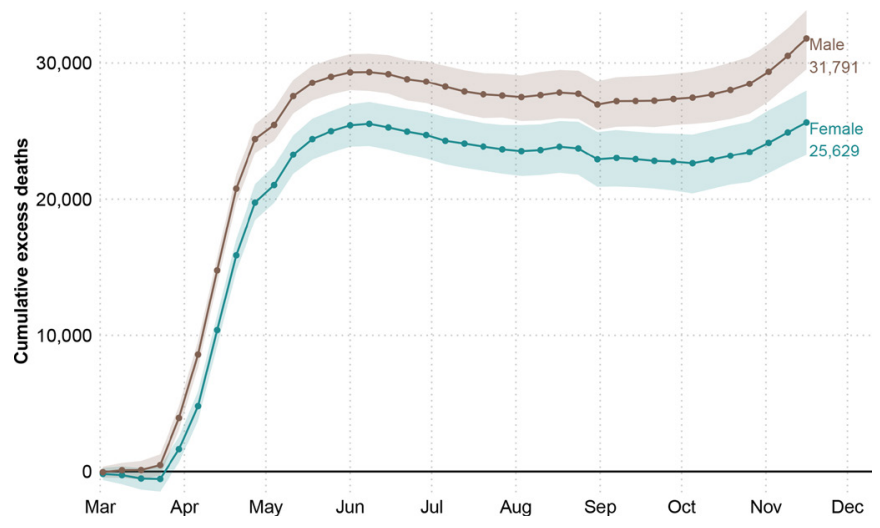

Figure 1 Cumulative excess deaths in England and Wales through the COVID-19 pandemic weeks $10-47$ of 2020 by sex. Shaded areas represent $95 \%$ prediction intervals. Excess deaths are defined as the total observed deaths subtracting the estimated baseline death count.

baseline (see figure 1). This estimate represents a 15.1\% (14.2, 16.2) increase in deaths compared with the expected level.

Death rates during the pandemic were consistently higher among men in all groups compared with women (see online supplemental figure 3). Male excess deaths accounted for 55.4\% (31 791 deaths) of total excess deaths, compared with $44.6 \%$ (25 629 deaths) among women over the same period, despite the fact that women make up a larger fraction of the older population. Between 2 March and 20 November, male deaths exceeded the expectation by $16.8 \%(15.4,18.0)$ and female death counts by $13.6 \%(12.2,14.9)$. Cumulative excess deaths at the end of the first wave (week 26 ending in June 29) were 53937 (95\% prediction interval: 53 092, 54 746) followed by no excess mortality over the summer months, before an uptick that started in October 2020 when a second wave emerged and excess deaths began to rise again.

Disaggregating by age, we estimate no excess deaths among those younger than 15 years. The 15-44-year-old age group accounted for $652(395,903)$ excess deaths $(6.2 \%(3.6,8.7)$ above the expected level). For older age groups, excess deaths rose sharply (see figure 2). The toll of the pandemic resulted in $7859(7065,8645)$ and $9835(8814,10833)$ excess deaths

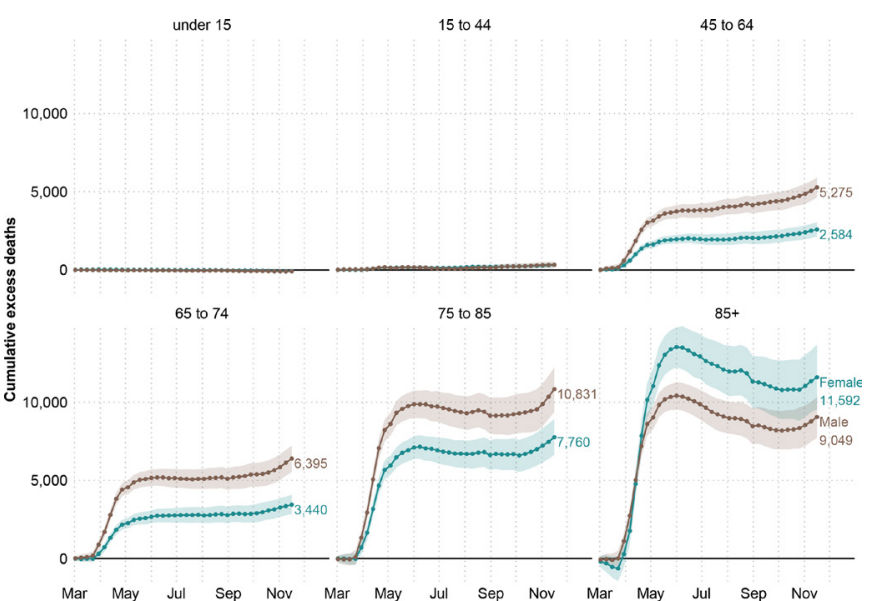

Figure 2 Cumulative excess deaths in England and Wales through the COVID-19 pandemic weeks 10-47 of 2020 by sex and age groups. Shaded areas represent $95 \%$ prediction intervals. Excess deaths are defined as the total observed deaths subtracting the estimated baseline mortality. among people between $45-64$ and 64-74 years of age, respectively. These numbers are $17.6 \%(15.6,19.7)$ and $16.0 \%(14.1$, 17.9) above the baseline. The largest numbers of lives lost were estimated among the groups $75-85$ and 85 and older, with $17.2 \%(15.3,19.2)$ and $13.7 \%(11.9,15.4)$ more deaths than expected. Among the former, 18591 (16 845, 20 435), excess deaths were estimated, while among the oldest age group, there were 20641 (18 271, 22 916) deaths above the baseline. Note the larger number of female excess deaths in the $85+$ age group is due to there being 1.6 times more women in this age group compared with men. After a peak in excess deaths by June 2020, the $85+$ age group saw somewhat lower than baseline mortality over the summer months, before an increasing trend in excess deaths emerged again in the second wave from October 2020. In contrast, for all other age groups, mortality remained at baseline over the summer months.

\section{Estimates of life expectancy and lifespan inequality}

Female life expectancy at birth increased from $81.4(81.3,81.4)$ years in 2005 to $83.5(83.5,83.6)$ years in 2019 in England and Wales (see figure 3). Similarly, male life expectancy increased from $77.1(77.1,77.2)$ to $79.9(79.8,79.9)$ years in the same period. Using data from the first 47 weeks of 2020 yields an estimated life expectancy at birth of $82.6(82.5,82.6)$ and 78.7 $(78.6,78.7)$ for women and men, respectively, a reduction of 0.9 years for women and 1.2 years for men.

From 2005 to 2019, lifespan inequality declined slowly from $13.8(13.7,13.9)$ to $13.5(13.4,13.6)$ years for women and from $15.0(15.0,15.1)$ to $14.7(14.6,14.7)$ years for men (see figure 3). Over the first 47 weeks of 2020, we estimate that lifespan inequality fell sharply to $13.1(13.0,13.1)$ and 14.2 $(14.2,14.3)$ years for women and men, respectively, corresponding to a reduction of nearly 5 months for both sexes.

\section{Sensitivity analysis}

We performed several sensitivity analyses. First, we refitted the seasonal baseline without including the first 9 weeks of 2020 . This adjustment did not have major effects on our estimates, and by taking the first 9 weeks into account, we aligned our predictions with the observed trend at the beginning of the year. Our four models produce central estimates of the number of excess deaths between 49056 and 57419 depending on the choice of the model and its assumptions, but do not substantively affect the pattern of our results. We note that excess deaths derived from the baselines estimated from both the generalised additive models and generalised linear models indicated a higher magnitude of excess deaths than those using average mortality rates from the preceding 5 years as the baseline. For full details, see online supplemental tables 1 and 2 . In addition, we also estimated life expectancy using a piecewise constant hazard model and the results did not change.

\section{DISCUSSION}

Excess deaths during the first 47 weeks of the year 2020 shed light on the cumulative burden of the COVID-19 pandemic in England and Wales. While several European countries have experienced substantially increased mortality over the course of the pandemic, data at hand suggest that England and Wales are among the worst performers in terms of excess deaths, especially in the working-age group $15-64 .{ }^{30}$ We estimated 57419 (54 197, 60 752) premature deaths due to the pandemic. Our estimate is based on a systematic comparison of different approaches to estimating a mortality baseline from which excess is derived and 


\section{A Life expectancy at birth}

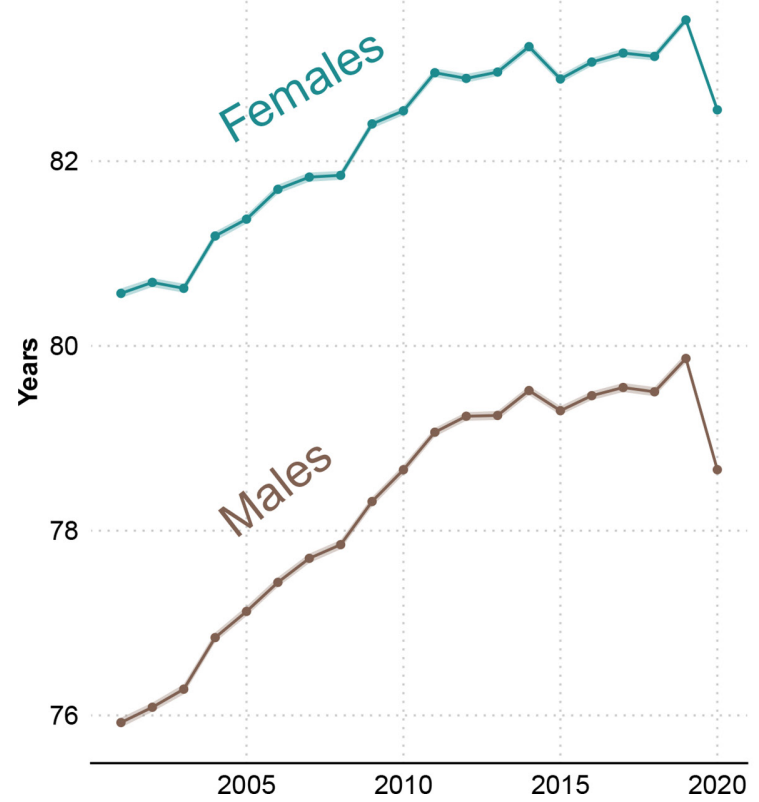

\section{B Lifespan inequality}
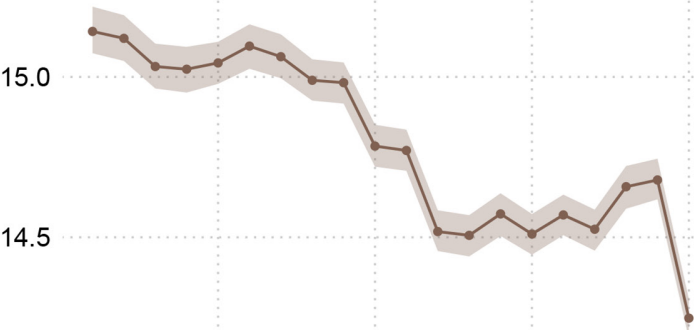

14.0

13.5

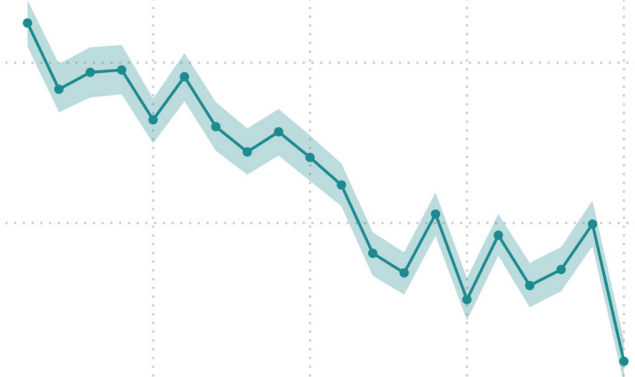

13.0

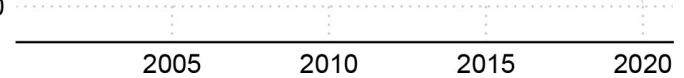

Figure 3 Life expectancy and lifespan inequality (SD of ages at death) estimates for the periods 2001-2019, and for 2020 considering the first 47 weeks of the year by sex. Shaded areas represent $95 \%$ prediction intervals.

relies on a refined model that accounts for changes in population ageing and seasonality. The toll of the pandemic had unequal impacts by age and sex in Europe and other regions. ${ }^{31-33}$ Similarly for England and Wales and consistent with other work, ${ }^{8}$ we found that excess mortality varied between sexes, with males accounting for $55 \%$ of excess deaths. Excess deaths increased sharply over age and male deaths were estimated to exceed women in all age groups, with the exception of those above age 85. This is explained by the population composition of England and Wales where more women survive to older ages. Accounting for this compositional effect, death rates during 2020 were higher among men in all ages groups (see online supplemental figure 3).

According to the ONS, between 1 March and 30 June 2020, there were 50335 deaths involving COVID-19, 46736 (93\%) of which assigned COVID-19 as the underlying cause of death based on information noted on the death certificate. ${ }^{21} \mathrm{~A}$ sizeable fraction of our estimate for excess deaths over the first wave of the pandemic is thus likely to be directly linked to COVID19. Based on preliminary cause of death analysis of other (nonCOVID) causes by the ONS, deaths occurring from Alzheimer disease and dementia, ischaemic heart disease, cerebrovascular diseases, influenza and pneumonia and 'symptoms signs and illdefined conditions' category were all higher between March and May 2020. ${ }^{22}$ Together Alzheimer and 'symptom signs and illdefined conditions' experienced the largest increases in magnitudes compared with previous years, and deaths occurring from asthma and diabetes at home also increased. ${ }^{22}$ These preliminary cause-of-death patterns suggest that a significant fraction of the unexplained excess mortality over the first wave of the pandemic may also be attributable to undiagnosed COVID-19. As more detailed cause-of-death data become available over the coming months, future research should seek to develop methods to disentangle excess deaths attributable to COVID-19 versus those arising indirectly due to effects such as reduced care for other conditions.
For the latter half of 2020, in the period from 15 June to the end of August (weeks 25-36), our estimates showed no excess mortality in most weeks for those under 85 before the emergence of a second wave of excess mortality from October. The lower than baseline mortality observed in the summer months of the $85+$ age group suggests potential mortality displacement effects, that is, that some deaths were brought forward in this age group due to the pandemic, although the numbers far from compensated for first wave deaths even at these oldest ages. However, no similar signs of mortality displacement due to lower than baseline mortality were visible for the other age groups over the summer. As these estimates are based on deaths registered so far, it is too early to clarify the contributions of mortality displacement to excess mortality observed during the pandemic and its impacts on postcrisis mortality levels.

Life expectancy in England and Wales had been steadily improving for 50 years before stagnating in the past decade. ${ }^{3435}$ We have provided estimates of life expectancy for 2019 and 2020 based on mortality data until week 47 , which show that life expectancy dropped a staggering 0.9 and 1.2 years for women and men, respectively, between these years. Moreover, our estimates for life expectancy fall 0.7 and 1.1 years below the official projected life expectancy in 2020 for women and men, ${ }^{36}$ respectively. To put this into perspective, male and female life expectancy regressed to the levels of 2010. It is likely that our estimates of excess deaths and life expectancy losses until this period are underestimated, as these estimates are based on deaths registered so far, a small fraction of which may have experienced registration delays. ${ }^{21}$ Our estimates also do not account for excess mortality that occurred during weeks $48-52$ of 2020, which are likely to further reduce life expectancy estimates for the full year. Recent evidence suggests that reversals and stagnation in life expectancy among developed countries are usually a result of mid-life mortality crises. ${ }^{35}$ In contrast, life expectancy losses during the pandemic have come about from sharp increases in older age mortality in both sexes. 
Historically, life expectancy increases have been accompanied by reductions in lifespan inequality due to mortality improvements at younger ages, although more recent studies have found that life expectancy improvements can occur even without accompanying reductions in lifespan inequality. ${ }^{13}$ Our results strikingly show a third, previously undocumented pattern of life expectancy and lifespan inequality change, with both decreasing concurrently due to the unique nature of the mortality stress triggered by the COVID-19 pandemic. In contrast with previous influenza pandemics such as the 1918-1920 Spanish influenza that primarily affected the young, ${ }^{37}$ or the 1957 pandemic that affected both the young and old, ${ }^{38}$ the mortality impact of the COVID-19 pandemic has primarily affected older age groups. Within a broader context of population health in which mortality is now largely concentrated at older ages, the elevated excess death rates at older age groups observed during the COVID-19 pandemic so far has reduced life expectancy. However, the disproportionate shift in the distribution of ages at death to older age groups made ages at death more similar, thereby reducing variation but at the expense of increasing overall average mortality. As a result of these dynamics, life expectancy and lifespan inequality moved in the same direction.

Looking forward, it is unclear if life expectancy will return to baseline levels rapidly, and even if/when it recovers, how mortality will be different. The prospect of vaccination being likely in the near future suggests a potential for the rapid recovery of life expectancy, although this will depend on the rollout speed, coverage and efficacy of the vaccine. ${ }^{39}$ In contrast,

\section{What is already known on this topic}

- COVID-19-related deaths may be misclassified, thereby inaccurately estimating the full impact of the pandemic on mortality. The pandemic may also have indirect effects on other causes due to changed behaviours as well as the social and economic consequences resulting from its management. Excess mortality, the difference between observed deaths and what would have been expected in the absence of the pandemic, is a useful metric to quantify the overall impact of the pandemic on mortality and population health. Life expectancy at birth and lifespan inequality assess the cumulative impact of the pandemic on population health.

\section{What this study adds}

- We examine death registration data from the Office for National Statistics from 2010 to week 47 (ending on 20 November) in 2020 to quantify the impact of the COVID-19 pandemic on mortality in England and Wales thus far. We estimate excess mortality risk by age and sex and quantify the impact of excess mortality risk on excess deaths, life expectancy and lifespan inequality. During weeks 10 to 47 of 2020, elevated mortality rates resulted in 57419 additional deaths compared with baseline mortality. Life expectancy at birth for women and men over the 47 weeks of 2020 was 82.6 and 78.7 years, with 0.9 and 1.2 years of life lost relative to the year 2019. Lifespan inequality, a measure of the spread or variation in ages at death, declined due to the increased mortality at older ages. the combination of potential scarring effects of COVID-19, such as the long-term consequences of the disease on individuals' health ${ }^{40}$ the implications of lockdown and non-pharmaceutical interventions on behaviours and mental health, ${ }^{41}$ cancer treatment delay associated with increased mortality ${ }^{42}$ and the unequal impact of COVID-19 across subgroups by age, sex, ethnicity socioeconomic status and regions, ${ }^{43}$ could create an unseen mortality profile that maintains life expectancy at lower levels beyond the short term into the medium term.

Twitter Jose Manuel Aburto @jm_aburto, Ridhi Kashyap @ridhikash07, Colin Angus@victimofmaths, Melinda C Mills @melindacmills and Jennifer Beam Dowd @ drjenndowd

Contributors JMA, RK, JS and JBD contributed to the design of the study. JMA RK and CA drafted the manuscript. JS and JMA performed the statistical analysis. All authors contributed to interpretation of data, revised the article critically for important intellectual content, and approved the final version of the manuscript. JMA and RK, the corresponding authors, attest that all listed authors meet authorship criteria and that no others meeting the criteria have been omitted.

Funding JMA and RK acknowledge support from the British Academy Newton International Fellowship; JMA and JS acknowledge support from the Rockwool Foundation grant on Excess Deaths; JMA, RK, JBD and MCM were supported by Leverhulme Trust, John Fell Fund and H2020 European Research Council Advanced Grant 835 079. CA was supported by the SIPHER Consortium (https://sipher.ac.uk/), part of the UK Prevention Research Partnership (MR/S037578/1), an initiative funded by UK Research and Innovation Councils, the Department of Health and Social Care (England) and the UK devolved administrations, and leading health research charities. Weblink: https://ukprp.org/

Competing interests None declared.

Patient consent for publication Not required.

Provenance and peer review Not commissioned; externally peer reviewed.

Data availability statement Data are available in a public, open access repository. All data and code used for this article is available in an open access repository (https://github.com/OxfordDemSci/Excess-Deaths). The repository includes aggregated death counts used in this paper retrieved from the ONS form https:// www.ons.gov.uk/peoplepopulationandcommunity/birthsdeathsandmarriages/deaths/ datasets/weeklyprovisionalfiguresondeathsregisteredinenglandandwales. The data and code are accessible for public use.

Supplemental material This content has been supplied by the author(s). It has not been vetted by BMJ Publishing Group Limited (BMJ) and may not have been peer-reviewed. Any opinions or recommendations discussed are solely those of the author(s) and are not endorsed by BMJ. BMJ disclaims all liability and responsibility arising from any reliance placed on the content. Where the content includes any translated material, BMJ does not warrant the accuracy and reliability of the translations (including but not limited to local regulations, clinical guidelines, terminology, drug names and drug dosages), and is not responsible for any error and/or omissions arising from translation and adaptation or otherwise.

Open access This is an open access article distributed in accordance with the Creative Commons Attribution 4.0 Unported (CC BY 4.0) license, which permits others to copy, redistribute, remix, transform and build upon this work for any purpose, provided the original work is properly cited, a link to the licence is given, and indication of whether changes were made. See: https://creativecommons.org/ licenses/by/4.0/.

\section{ORCID iDs}

Jose Manuel Aburto http://orcid.org/0000-0002-2926-6879

Ridhi Kashyap http://orcid.org/0000-0003-0615-2868

Jonas Schöley http://orcid.org/0000-0002-3340-8518

Colin Angus http://orcid.org/0000-0003-0529-4135

John Ermisch http://orcid.org/0000-0002-3633-5246

Melinda C Mills http://orcid.org/0000-0003-1704-0001

Jennifer Beam Dowd http://orcid.org/0000-0003-2006-5656

\section{REFERENCES}

1 Weinberger DM, Chen J, Cohen T, et al. Estimation of excess deaths associated with the COVID-19 pandemic in the United States, March to may 2020. JAMA Intern Med 2020;180:1336-44

2 Li R, Pei S, Chen B, et al. Substantial undocumented infection facilitates the rapid dissemination of novel coronavirus (SARS-CoV-2). Science 2020;368:489-93.

3 ONS. The different uses of figures on deaths related to COVID-19 published by DHSC and the ONS - Office for National Statistics. Available: https://www.ons.gov.uk/news/ 
statementsandletters/thedifferentusesoffiguresondeathsfromcovid19publishedbydhsca ndtheons [Accessed 3 Dec 2020].

4 Vandoros S. Excess mortality during the Covid-19 pandemic: early evidence from England and Wales. Soc Sci Med 2020;258:113101.

5 Vollmer M, Radhakrishnan S, Kont M. Report 29: the impact of the COVID-19 epidemic on all-cause attendances to emergency departments in two large London hospitals: an observational study, 2020.

6 Tillett HE, Smith JW, Gooch CD. Excess deaths attributable to influenza in England and Wales: age at death and certified cause. Int J Epidemiol 1983;12:344-52.

7 Darby SC, Rizza CR, Doll R, et al. Incidence of AIDS and excess of mortality associated with HIV in haemophiliacs in the United Kingdom: report on behalf of the directors of haemophilia centres in the United Kingdom. BMJ 1989;298:1064-8.

8 Kontopantelis E, Mamas MA, Deanfield J, et al. Excess mortality in England and Wales during the first wave of the COVID-19 pandemic. J Epidemiol Community Health 2021;75:213-23.

9 Office for National Statistics. Deaths registered Weekly in England and Wales, provisional: week ending 14 August 2020. Available: https://www.ons.gov.uk/peop lepopulationandcommunity/birthsdeathsandmarriages/deaths/bulletins/deathsregist eredweeklyinenglandandwalesprovisional/weekending14august2020 [Accessed 1 Sep 2020].

10 Dowd JB, Andriano L, Brazel DM, et al. Demographic science AIDS in understanding the spread and fatality rates of COVID-19. Proc Natl Acad Sci U SA 2020;117:9696-8.

11 Nepomuceno MR, Acosta E, Alburez-Gutierrez D, et al. Besides population age structure, health and other demographic factors can contribute to understanding the COVID-19 burden. Proc Natl Acad Sci U S A 2020;117:13881-3.

12 Preston S, Heuveline P, Guillot M. Demography, measuring and modeling population processes, 2002

13 Aburto JM, Villavicencio F, Basellini U, et al. Dynamics of life expectancy and life span equality. Proc Natl Acad Sci U S A 2020;117:5250-9.

14 van Raalte AA, Sasson I, Martikainen P. The case for monitoring life-span inequality. Science 2018;362:1002-4.

15 Edwards RD, Tuljapurkar S. Inequality in life spans and a new perspective on mortality convergence across Industrialized countries. Popul Dev Rev 2005;31:645-74.

16 Canudas-Romo V. The modal age at death and the shifting mortality hypothesis. Demogr Res 2008;19:1179-204.

17 ONS. All data related to Population estimates for the UK, England and Wales, Scotland and Northern Ireland: mid-2019 - Office for National Statistics. Available: https://www.ons.gov.uk/peoplepopulationandcommunity/populationandmigration/ populationestimates/bulletins/annualmidyearpopulationestimates/mid2019estimates/ relateddata [Accessed 10 Jul 2020].

18 ONS. National population projections - Office for National Statistics. Available: https:// www.ons.gov.uk/peoplepopulationandcommunity/populationandmigration/populati onprojections/bulletins/nationalpopulationprojections/2018based [Accessed $10 \mathrm{Jul}$ 2020].

19 Dougherty RL, Edelman AS, Hyman JM. Nonnegativity-, monotonicity-, or convexitypreserving cubic and quintic Hermite interpolation. Math Comput 1989;52:471-94.

20 ONS. Deaths registered in England and Wales - Office for National Statistics. Available: https://www.ons.gov.uk/peoplepopulationandcommunity/birthsdeathsandmarriages/ deaths/datasets/deathsregisteredinenglandandwalesseriesdrreferencetables [Accessed 10 Jul 2020].

21 ONS. Deaths involving COVID-19, England and Wales - Office for National Statistics. Available: https://www.ons.gov.uk/peoplepopulationandcommunity/birthsdeaths andmarriages/deaths/bulletins/deathsinvolvingcovid19englandandwales/deathsoccurr inginmay2020\#time-taken-for-the-deaths-in-march-april-and-may-to-be-registered [Accessed 14 Jul 2020].

22 ONS. Analysis of death registrations not involving coronavirus (COVID-19), England and Wales - Office for National Statistics. Available: https://www.ons.gov.uk/peoplepo pulationandcommunity/birthsdeathsandmarriages/deaths/articles/analysisofdeath egistrationsnotinvolvingcoronaviruscovid19englandandwales28december2019to $1 \mathrm{ma}$ y2020/technicalannex [Accessed 14 Jul 2020].

23 ONS. Impact of registration delays on mortality statistics in England and Wales - Office for National Statistics. Available: https://www.ons.gov.uk/peoplepopulationandc ommunity/birthsdeathsandmarriages/deaths/articles/impactofregistrationdelaysonmort alitystatisticsinenglandandwales/2018 [Accessed 10 Jul 2020].

24 Wood SN. Generalized additive models: an introduction with R. Second ed. CRC Press, 2017.

25 Nielsen J, Krause TG, Mølbak K. Influenza-Associated mortality determined from allcause mortality, Denmark 2010/11-2016/17: the FluMOMO model. Influenza Other Respir Viruses 2018;12:591-604.

26 Serfling RE. Methods for current statistical analysis of excess pneumonia-influenza deaths. Public Health Rep 1963;78:494-506.

27 Andreev EM, Shkolnikov VM. Spreadsheet for calculation of confidence limits for any life table or healthy-life table quantity. Working paper Max Planck Institute for Demographic Research, 2010.

28 Team RC. R: a language and environment for statistical computing. Vienna, Austria, 2013.

29 Aburto JM, Schoeley J. OxfordDemSci/Excess-Deaths. Leverhulme centre for demographic science, 2020. Available: https://github.com/OxfordDemSci/ExcessDeaths [Accessed 10 Jul 2020].

30 A pandemic primer on excess mortality statistics and their comparability across countries. our world data. Available: https://ourworldindata.org/covid-excess-mortality [Accessed 9 Jul 2020].

31 Dowd JB, Andriano L, Brazel DM, et al. Demographic science AIDS in understanding the spread and fatality rates of COVID-19. Proc Natl Acad Sci U SA

32 Verity R, Okell LC, Dorigatti I, et al. Estimates of the severity of coronavirus disease 2019: a model-based analysis. Lancet Infect Dis 2020;20:669-77.

33 Kashnitsky I, Aburto JM. COVID-19 in unequally ageing European regions. World Dev 2020;136:105170.

34 Hiam L, Harrison D, McKee M, et al. Why is life expectancy in England and Wales 'stalling'? J Epidemiol Community Health 2018;72:404-8.

$35 \mathrm{JY} \mathrm{H}$, Hendi AS. Recent trends in life expectancy across high income countries: retrospective observational study. BMJ2018:362

36 ONS. Life tables, principal projection, England and Wales - Office for National Statistics. Available: https://www.ons.gov.uk/peoplepopulationandcommunity/birt hsdeathsandmarriages/lifeexpectancies/datasets/lifetablesprincipalprojectionengland andwales [Accessed 14 Jul 2020].

37 Gagnon A, Miller MS, Hallman SA, et al. Age-Specific mortality during the 1918 influenza pandemic: unravelling the mystery of high young adult mortality. PLoS One 2013:8:e69586.

38 Viboud C, Simonsen L, Fuentes R, et al. Global mortality impact of the 1957-1959 influenza pandemic. J Infect Dis 2016:213:738-45.

39 Mills M, Rahal C, Brazel DM. COVID-19 vaccine deployment: behaviour, ethics, misinformation and policy strategies. Available: https://royalsociety.org/news/2020/ 11/vaccine-hesitancy-threatens-to-undermine-pandemic-response/ [Accessed $26 \mathrm{Nov}$ 2020].

40 Mahase E. Covid-19: What do we know about "long covid" ? BMJ 2020;370.

$41 \mathrm{Xu}$ X, Banks J. The mental health effects of the first two months of lockdown and social distancing during the Covid-19 pandemic in the UK. The IFS, 2020.

42 Hanna TP, King WD, Thibodeau S. Mortality due to cancer treatment delay: systematic review and meta-analysis. BMJ2020;371.

43 Bambra C, Riordan R, Ford J, et al. The COVID-19 pandemic and health inequalities. J Epidemiol Community Health 2020;74:964-8.

44 Trias-Llimós S, Riffe T, Bilal U. Monitoring life expectancy levels during the COVID-19 pandemic: example of the unequal impact of the first wave on Spanish regions. PLoS One 2020;15:e0241952. 